\title{
Improved Order Morphology Transforms and Its Application in the Edge Detection of Circular Impact Craters
}

\author{
Yuanni Wang ${ }^{1 *}$ and Hong Cao ${ }^{2}$ \\ ${ }^{1}$ School of Computer of China University of Geosciences, whan, 430074, china \\ ${ }^{2}$ School of Computer of China University of Geosciences, wuhan, 430074, china \\ 1ynwang2005@163.com,2percy2007@163.com
}

\begin{abstract}
Because of the existence of the noise, edge blur and fracture or overlap, there are still some difficulties of the edge extraction of the lunar image craters. For this, the circular impact craters as an example, this paper has built an extraction model based on an improved order morphological transform, for the better image denoising, complete details, and the edge detection more accurate. The experimental results show the edge detection model of the order morphological transformation of the edge information entropy weighted is better by comparing with the common edge detection operator and the morphological operator of the anti-noise type. In addition, this paper compares the information entropy of the different edge extracted methods. From the effect and the result of the information entropy Figures, it can be seen, the model can effectively extract the crater edge. The experimental results show the validity of the model, and successfully applied to the edge extraction of the lunar crater image.
\end{abstract}

Keywords: Impact craters; Edge extraction; Order morphology; Morphology transformation

\section{Introduction}

Order morphology transformation theory introduced the statistical methods based on the morphology and the edge detection based on order morphology transformation has obvious advantages for noisy image [1]. People can acquire the optimum image effect through choosing the appropriate morphology arithmetic parameters according to actual background, which are mainly the form and dimension of the structure element, the value of the percentile and its compound form shift.

For the lunar images, extracting the topographical features of interest, such as impact craters, can help to analyze the morphological characteristics of the lunar surface, and provide some important references for the research of the lunar surface terrain characteristics and material composition. It is of great significance for the exploration of the moon to study how to extract the object of interest from lunar images. At present, the extraction of lunar images is divided into two major categories, one is the detection of the surface of the moon; another is the detection of the impact crater. Burl, Honda, and etc. have made a number of automatic and semi-automatic crater detection methods [2] since 2001, but the accuracy is not high, which can't be applied to reality. When the craters edge blur, fracture or overlapping, and noise contained, there are still some difficulties to automatically detect the crater. Sawabe presented multiple methods to detect the impact craters for Apollo and Clementine image [3]. Some researchers have also made some extraction method crater [4-6], and achieved craters extraction in some particular cases.

Although there are some scholars researching the lunar image craters extraction, but there are still many issues to be resolved, for example, data accuracy and adaptability to different methods. The recognition accuracy still needs to be further improved. Especially 
for multi-scale, complex and diverse impact craters, such as multi-ring crater, superimposed craters and so on.

This paper has improved the order morphology transformation, in order to better maintain the integrity and details of the image in the improvement of the image denoising ability. At the same time, use the improved method for the edge extraction of the ring craters.

\section{Morphological Transformation}

\subsection{Morphological Transformation Ideas}

Mathematical morphology is a nonlinear filtering method. It can reflect the image collections characteristics of the edge detection, a better image edge detection, but also to meet real-time requirements and solve the coordination problem of edge detection precision and anti-noise performance. Compared with traditional algorithms, morphology has unique advantages in the image edge detection.

Morphological edge detection operators contain dilation, erosion, opening, closing and various combinations through four operations. If $\mathrm{f}(\mathrm{n})$ represents the image function, $\mathrm{B}$ as the structural element, $\mathrm{f}$ ' as the result of edge detection function, the basic morphological edge detection operators [7-9] as follows:

$$
\begin{gathered}
\text { dilation: } f^{\prime}=f(n) \oplus B-f(n) \\
\text { erosion: } f^{\prime}=f(n)-f(n) \Theta B \\
\text { dilation erosion: } f^{\prime}=f(n) \oplus B-f(n) \Theta B \\
\text { opening: } f^{\prime}=f(n)-f(n) \circ B \\
\text { closing: } f^{\prime}=f(n) \bullet B-f(n) \\
\text { opening and closing: } f^{\prime}=f(n) \bullet B-f(n) \circ B
\end{gathered}
$$

Use the characteristics of expansion, corrosion, and opening and closing operation, to improve three basic operators of above latter, get anti-noise type morphological edge detection operator [10] as follows:

$$
\begin{aligned}
& \text { anti-noise dilation: } f^{\prime}=f(n) \oplus B-f(n) \bullet B \\
& \text { anti-noise erosion: } f^{\prime}=f(n) \circ B-f(n) \Theta B \\
& \text { anti-noise dilation and erosion: } \quad f^{\prime}=(f(n) \circ B) \oplus B-(f(n) \bullet B \Theta B
\end{aligned}
$$

\subsection{Order Morphology Transformation}

Order morphology transformation theory is to introduce a statistical approach on the basis of morphology, and it has obvious advantages for the image noise. There are several definitions of the order morphology transformation of the discrete form as follows [11]:

Definition 1

As $f(x)$ defined the discrete function of the $\mathrm{n}$-dimensional discrete data space $Z^{(n)}, B=\left\{x_{1}, x_{2}, \ldots, x_{n}\right\}$ as the structural elements, the measure ${ }^{\mu(\square)}$ as the point count satisfied $0<\mu(B)=N<+\infty$, the $\mathrm{N}$ values of $f(x)$ in the $\mathrm{B}$ on ordered from small to large $f\left(x_{1}{ }^{\prime}\right) \leq f\left(x_{2}{ }^{\prime}\right) \leq \ldots \leq f\left(x_{N}{ }^{\prime}\right)$, so, $f(x)$ of $\mathrm{D}$ rank order in $\mathrm{B}$ is defined as $\operatorname{ord}\{d, f \mid B\} \square f\left(x_{d}{ }^{\prime}\right), d=1,2, \ldots, N$.

\section{Definition 2}

The order morphology transformation of the discrete function $f(x)$ about the structural elements of $\mathrm{B}$ is $f_{\subsetneq B} B$ defined as

$$
\begin{aligned}
& \left(f_{Ф} B\right)(x)=\operatorname{ord}\left\{d, f \mid B_{x}^{\vee}\right\} \\
& =\operatorname{ord}\left\{(N-1) p+1 ; f \mid B_{x}^{\vee}\right\}
\end{aligned}
$$


Above the definition, $\mathrm{p}=0,1 /(\mathrm{N}-1), \ldots, 1, B_{x}^{\vee}=\{x-b: b \in B\}, d=(N-1) p+1, \quad \mathrm{p}$ is the percentile of the order morphology transformation. ${ }^{p=\frac{d-1}{N-1}}$, in particular, when $\mathrm{p}=0,0.5,1,{ }^{f} \mathrm{p} B$ respectively, as the corrosion, median and expansion operations.

It has an advantage in the noise image of order morphology transformation edge detection. It can be combined with the actual application background, through the proper selection of morphological parameters to get the best effect of image processing, is mainly the shape and size of structure element of morphological operations, the value of percentile and the composite mode of percentile morphological transform [1].

\section{Model Establishment of Polycyclic Impact Craters Extraction Based on Improved Order Morphology Transformation}

\subsection{Algorithm}

For the morphological transformation, the noise removal results are good of opening and closing operation, but in the edge extraction, the opening and closing operations information is loss seriously, less clear than the extraction of the dilation and erosion operations. Compared to the operators of anti-noise dilation, anti- erosion, anti- noise dilation and erosion for morphological edge detection, it changes finding the maximum and minimum values of the dilation and erosion operations to the percentile of $\mathrm{p}$, increasing its operational flexibility, determine the transformation to the original image through the values of control $\mathrm{p}$, the changes also includes cases of doing dilation and erosion.

This paper introduces the edge information entropy to guide the edge detection of the anti-noise order morphological transform, and to establish an order morphological transform edge detection model based on information entropy weight, for improving the signal-to-noise ratio and accuracy. Control the three anti- noise edge detection weight by the edge information entropy, and at the same time introduce different directions structural elements to match the diversity of the edge, improving the traditional mathematical morphology edge detector, so as to improve the positioning accuracy, and restrain the noise better. If the edge result doesn't get a single pixel, then refine it.

The specific implementation steps are as follows:

STEP1: Initialize parameters of the percentile $\mathrm{p}$ and $\mathrm{q}$, and the structural element $\mathrm{B}$.

STEP2: Use the model established for the edge extraction.

STEP3: Thin edge extracted.

STEP4: Output the edge results.

\subsection{Model Established}

The research has shown that the better image edge extraction can be got by the antinoise order morphology transformation [12], on the basis to the further improvement, comprehensive consideration of the three kinds of anti-noise order morphology transform to the edge extraction model, we introduce edge information entropy to guide the weight factors of the anti-noise order morphology transformations, then establish the model of the weighted order morphology transformation based on the edge information entropy. This formula is as follows:

$$
\begin{aligned}
& f^{\prime}=\sum_{i=1}^{3} H_{i} f_{i}=P_{i} \lg \left(1 / P_{i}\right) f_{i}=-P_{i} \lg \left(P_{i}\right) f_{i}
\end{aligned}
$$

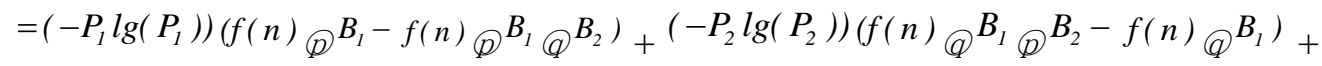

$$
\begin{aligned}
& \left(-P_{3} \lg \left(P_{3}\right)\right)\left(f(n) \oplus^{B_{1}} \emptyset^{B_{2}} \emptyset^{B_{3}-f(n)} \oplus^{B_{1}} \oplus^{B_{2}} \oplus^{B_{3}}\right)
\end{aligned}
$$


In the above formula, $\mathrm{H}_{\mathrm{i}}$ as the edge information entropy of a kind of order morphology transformation, $\mathrm{P}_{\mathrm{i}}$ as the edge probabilities, $\mathrm{fi}$ as the corresponding edge extraction results. Here, $f_{i}$ is corresponding to the three kinds of anti-noise order morphology transformation [12].

\subsection{Parameters Design}

Considering the selection of structural elements in the scale and the direction, this paper chooses two scales of $3 \times 3$ and $5 \times 5$, mainly to take into account the horizontal direction, the diagonal direction and the vertical direction.

In three kinds of anti-noise order morphological edge models, the structure element has been used orderly as the following structural elements: $\left[\begin{array}{llllllllll}1 & 0 & 1 & 0 & 1 & 0 & 1 & 1 & 1\end{array}\right], \quad\left[\begin{array}{llllllll}1 & 1 & 1 & 0 & 1 & 1 & 1\end{array}\right.$

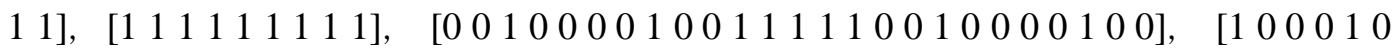
$101000100011010100001]$.

\section{Experiments}

(1)Detection of the order morphology transformation based on edge information entropy weighted

This paper has extracted the edge by the order morphological edge detection model based on the information entropy weighted. Figure 2 gives the edge detection effect with $\mathrm{p}$ as $1 / 3$.

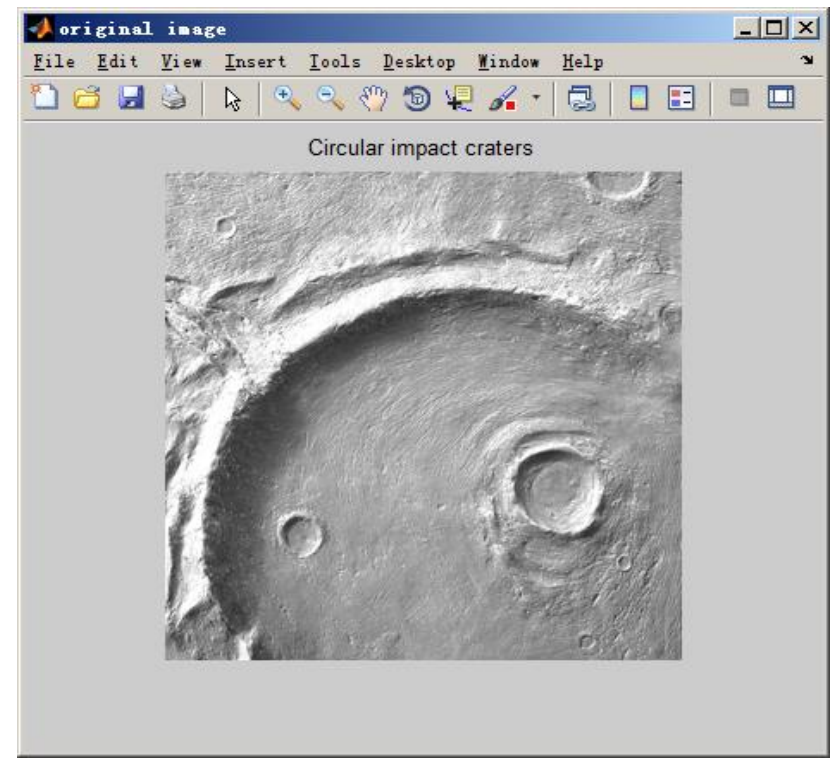

Figure 1. Circular Impact Craters 


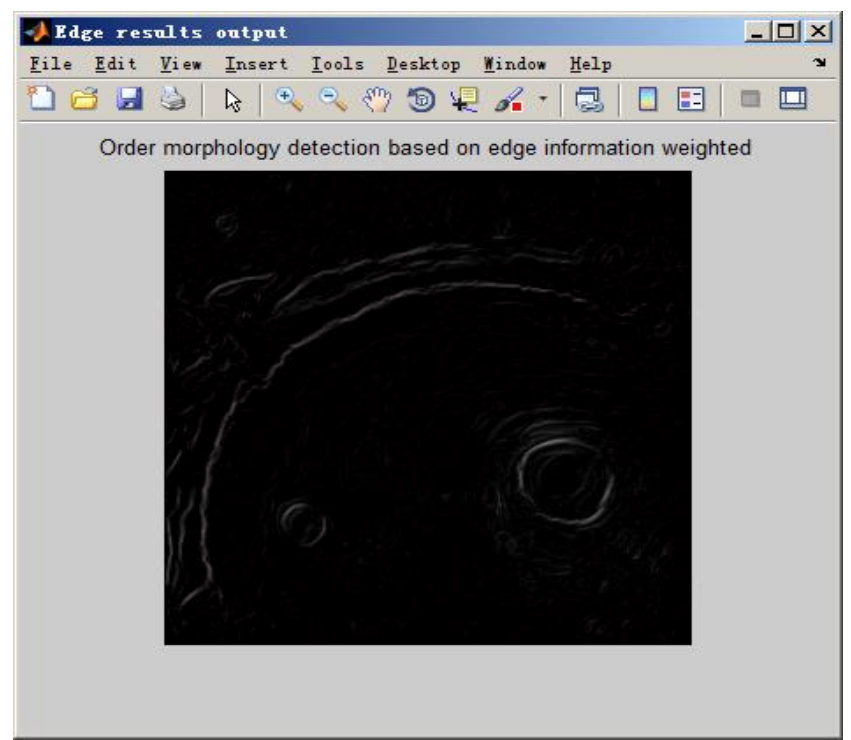

Figure 2. Order Morphology Detection Based on Edge Information Weighted

From the Figure 2, it can be seen that the edge of the impact crater can be extracted more accurately, and the edge is complete.

(2) Compared with canny operator, wavelet and anti-noise morphological transform operator

Figure 1 as an example, use canny operator and anti-noise morphological edge detection operator to extract the edge, the effect diagrams as follows.

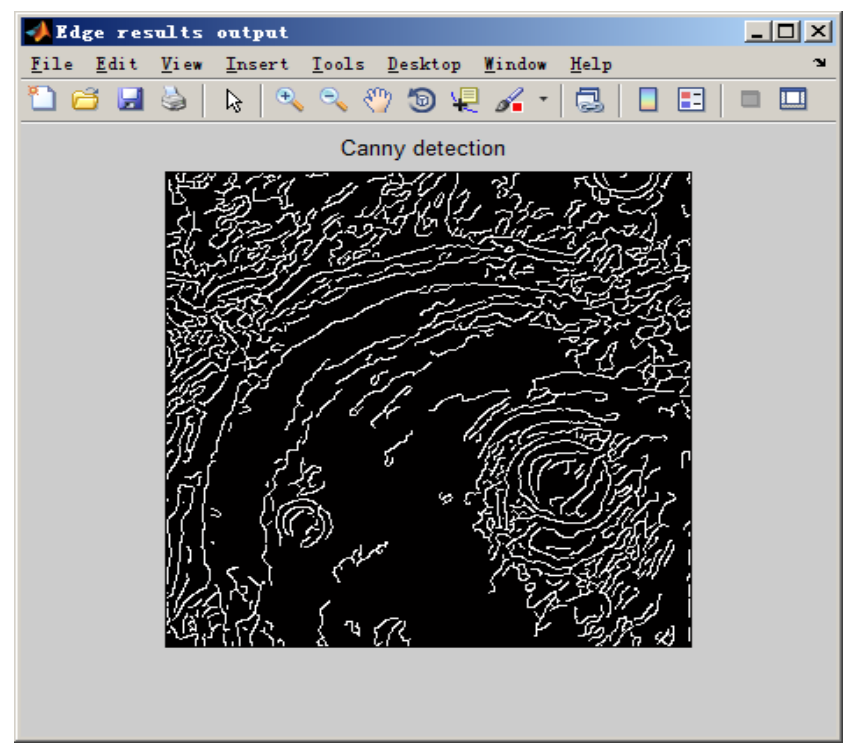

Figure 3. Canny Detection

From the Figure 3, it can be seen that the detection effect of canny operator is not satisfactory, and it shows too many small regions. 


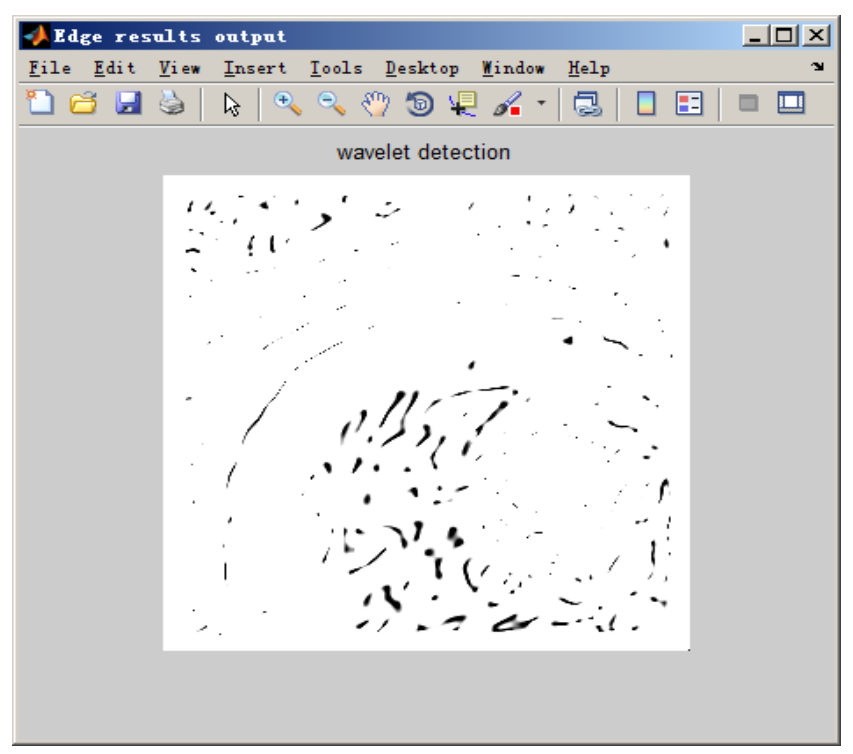

Figure 4. Wavelet Detection

In the Figure 4, the effect of detection is not good, too. It isn't included the edge of two important circular craters and the largest crater edge outer-ring is not clear, fracture.

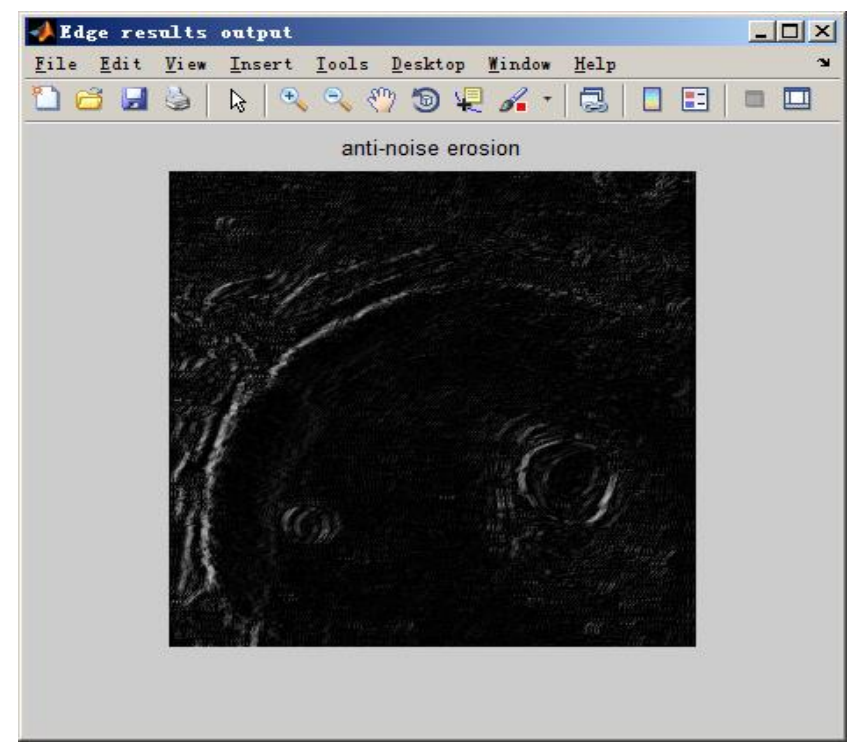

Figure 5. Anti-Noise Erosion 


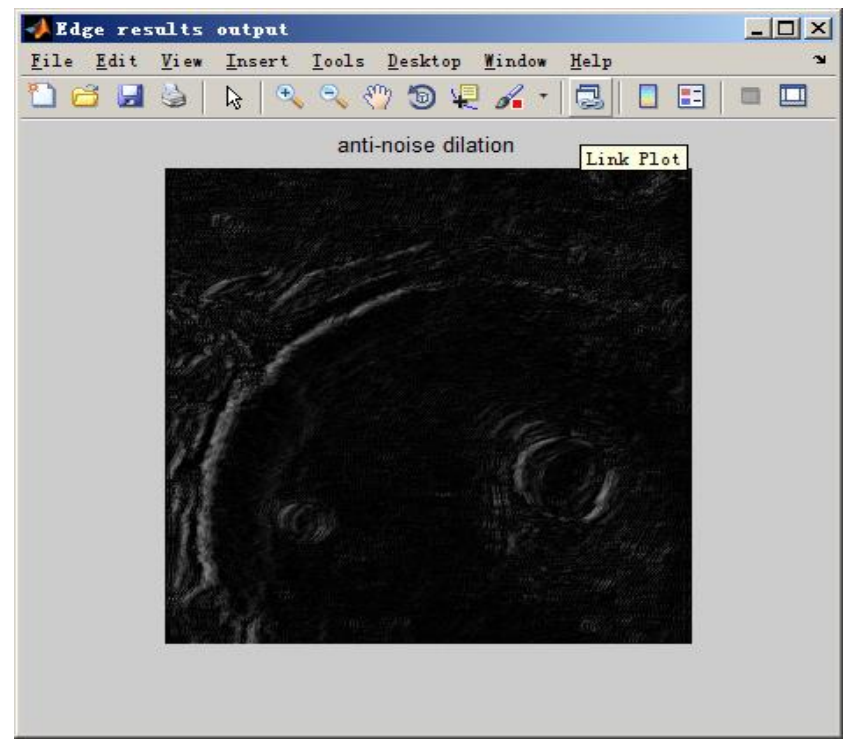

Figure 6. Anti-Noise Dilation

From the Figure 5 to Figure 6, it can be seen, relative to canny operator, the edge extraction based on the operators of anti-noise morphology transforms is relatively accurate, not too many fragments of the regional, the disadvantage is slightly fuzzy edge, and there are a few extra small edges.

From the Figure 3 to Figure 7, it can be seen, the order morphological edge detection of information entropy weighted relatively accurate, compared with the canny operator and wavelet, is not too much fragmented region; relative to effect of the general anti-noise morphological transform operator, edge sharpness is relatively high, and the redundant small edge is reduced relatively.

By comparing with the common edge detection operator and the morphological operator of the anti- noise type, the edge detection of the order morphological transformation of the edge information entropy weighted is better.

(3) Performance evaluation

In addition, in order to compare the effect of different methods, this paper compares the information entropy of the different edge extracted methods. The model established with the edge information entropy weighted has more advantages. The comparison is in the Figure below.

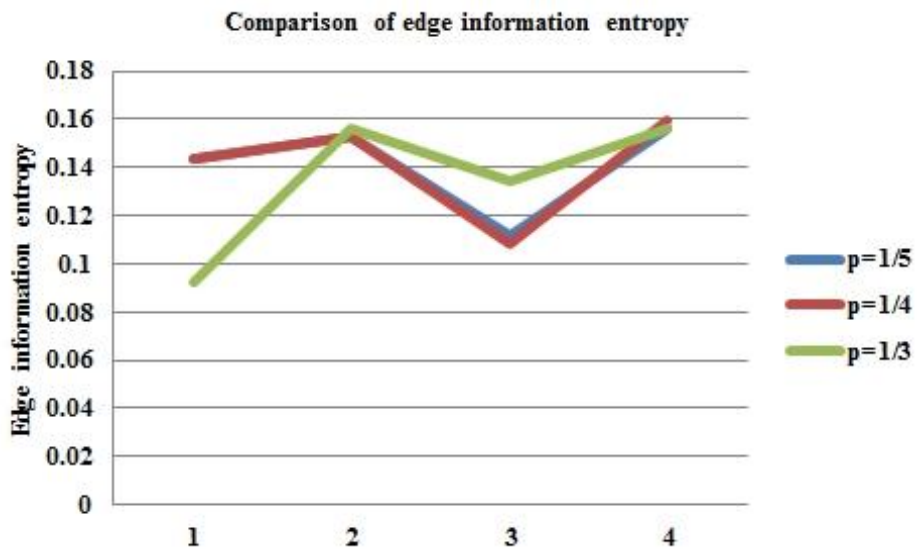

Figure 7. Comparison of Edge Information Entropy of Four Model of Edge Extraction 
In the above Figure, we compare the edge information entropy of the order morphology transformation model based on the edge information entropy weighted built in this paper with the order morphology models of anti- noise dilation, anti- noise erosion and anti- noise dilation and erosion, respectively in three cases of $p=1 / 5, p=1 / 4, p=1 / 3$. In the graph, the abscissa 1-4 corresponding to four kinds of extraction methods, 1-3 as the corresponding types of the order morphology anti-noise dilation, anti-noise erosion and anti-noise dilation and erosion, 4 as the order morphology transform based on the edge information entropy weighted. In each case the fluctuation in the abscissa of 2 and 4 are small, good stability, obviously the information entropy in the abscissa 4 always higher than that in the abscissa 2 . That is to say the stability of the order morphology transform based on the edge information entropy weighted is good, not affected with the change of $\mathrm{p}$, and the edge information entropy maximum. While there are big fluctuations in the horizontal ordinate 1 and 3, it demonstrates that the types of the order morphology anti-noise dilation and anti-noise dilation and erosion are influenced by the change of $\mathrm{p}$, and the stability is relatively poor.

(4) Concentric ring edge detection

Here, we have also carried on the edge extraction of the concentric rings, and the effects are in the Figures below.

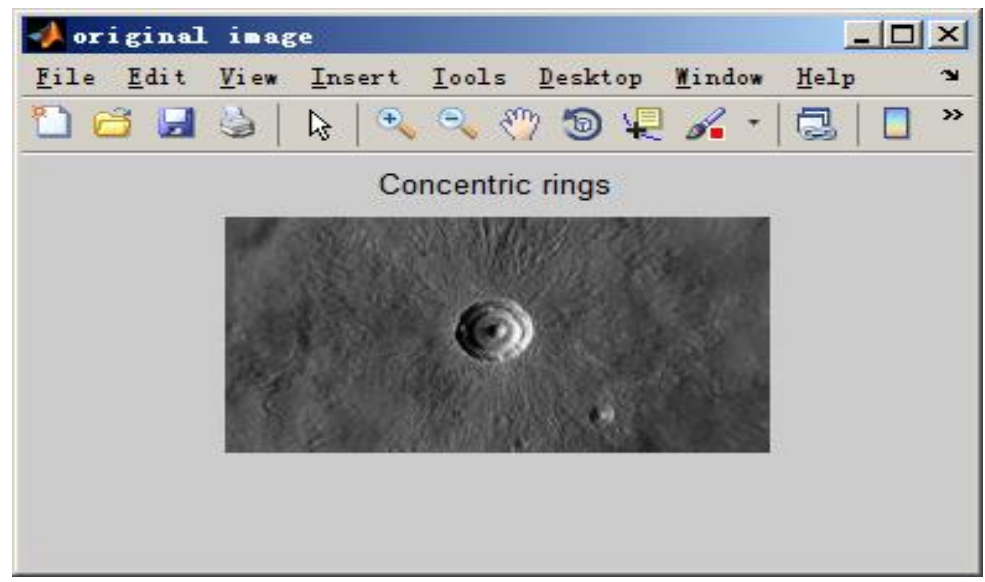

Figure 8. Concentric Rings

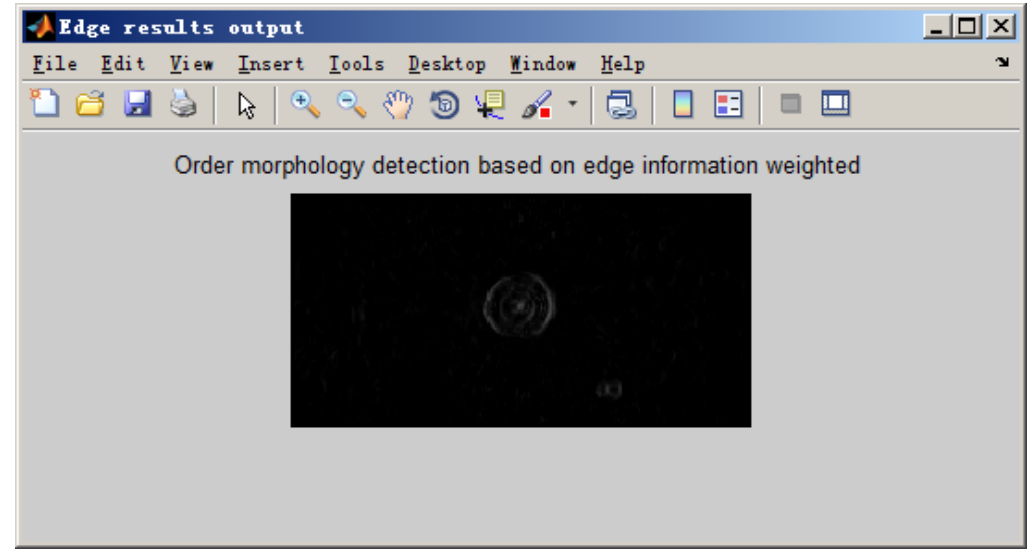

Figure 9. Edge Detection of Concentric Rings

From the effect and the result of the information entropy Figures above, it can be seen, for the ring structure, the model can effectively extract the crater edge. 


\section{Conclusions}

For the noise, edge blur and fracture or overlap of the lunar image craters, this paper has established a kind of order morphology transform edge detection model based on information entropy, on the base of order morphology transformation. It's for the better image denoising, complete details and the edge detection more accurate. Then, it applies to the edge extraction of the ring craters of the lunar image. As obvious multi-ring impact craters contained an example, the edge detection of the order morphological transformation of the edge information entropy weighted is better by comparing with the common edge detection operator and the morphological operator of the anti- noise type. In addition, in order to compare the effect of different methods, this paper compares the information entropy of the different edge extracted methods.

From the effect and the result of the information entropy Figures above, it can be seen, for the ring structure, the model can effectively extract the crater edge. Through the experiment data comparison, it verifies the stability of the method established, preserving the edge information, but also can detect the concentric ring. The experimental results show that this model is effective for the crater detection of the ring structure. The next step will be to consider the extracting of complex crater.

\section{Acknowledgment}

The authors would like to thank the Fundamental Research Funds for the National Natural Science Foundation of China (No.41202231) and the Central Universities, China University of Geosciences (Wuhan) (No.CUG100707 and No.CUG130106) for financially supporting this research.

\section{References}

[1] Wu Min-jin. Image morphology. Beijing: scientific and technical literature Publishing Press. (1990), pp. 214-229.

[2] Burl M C, Stough T, Colwell W, Automated detection of craters and other geological features. Proceedings of the 6th International Symposium on Artificial Intelligence and Robotics\&Automation in Space,Montreal,Canada, (2001) June 18-22.

[3] Y.Sawabe, T.Matsunaga, S.Rokugawa. Automated detection and classification of lunar craters using multiple approaches. Advances in Space Research. vol. 37, no. 1, (2006), pp. 21-27.

[4] Liu Yu-xuan, Liu Jian-jun, Mou Ling-li, A Review of Impact-Crater Detection. vol. 9, no. 2, (2012), pp. 203-209.

[5] Yuan Yue-feng, Zhu Pei-ming, Zhao Na, Automatic identification of circular mare craters based on mathematical morphology. Chinese Sci Sin-Phys Mech Astron, no. 43, (2013), pp. 324-332.

[6] Zhang Feng, Zhou Yong-liao,Zhen Yong-chun, A new automated approach to detecting and extracting lunar craters and its application. Earth Science Frontiers, vol. 19, no. 6, (2012), pp. 118-127.

[7] Cui Yi. Image processing and analysis-methods and application of mathematical morphology. Beijing: Science press,(2000).

[8] Serra J.Image analysis and mathematical morphology[M].London:Academic Press. 1982.

[9] Banghaln J A, Marshall S. Image and signal processing with mathematical morphology. Electronics\&Comrnunlcation Engineering Journal. vol. 3, no. 2, (1998), pp. 156-158.

[10] Yang Shu-bin, Peng Fu-yuan. Application of Morphological Edge Detectors in Image Corrupted by Noise. Computer engineering and applications. vol. 38, no. 17, (2002), pp. 91-92.

[11] ZHAO Chun-hui, HUI Jun-yin, WANG Wei, A Class of Adaptive Ranked-OrderMorphological Filters [J]. Journal of Image and Graphics, vol. 5, no. 8, (2000), pp. 674-677.

[12] Wang Yuan-ni, Ge Fei. Adaptive Order Morphology Edge Detection. Journal of computers, vol. 7, no. 4, (2012), pp. 846-850.

[13] WAN Cong, CHENG WeiMing, etc. Automatic extraction of lunar impact craters from Chang'E-1 satellite photographs. SCIENCE CHINA Physics, Mechanics \& Astronomy. vol. 55, no. 1, (2012), pp. 162-169.

[14] Hwang, Bo-Yeon; Jung, Jae-Hoon; Lee, Jang-Myung.Advanced sound source localization study using de-noising filter based on the discrete wavelet transform(DWT). Journal of Institute of Control, Robotics and Systems, vol.21, no. 12, (2015), pp. 1185-1192.

[15] Tu, Ke; Li, Hongbo ; Sun, Fuchun1.A statistical learning based image denoising approach. Frontiers of Computer Science, vol.9, no. 5, (2015), pp. 713-719. 
International Journal of Control and Automation Vol.10, No.4 (2017) 